\title{
Human pluripotent stem cell-derived brain organoids as in vitro models for studying neural disorders and cancer
}

\author{
Juan Luo and Peng Li
}

\begin{abstract}
The sheer complexities of brain and resource limitation of human brain tissue greatly hamper our understanding of the brain disorders and cancers. Recently developed three-dimensional (3D) brain organoids (BOs) are self-organized and spontaneously differentiated from human pluripotent stem cells (hPSCs) in vitro, which exhibit similar features with cell type diversity, structural organization, and functional connectivity as the developing human brain. Based on these characteristics, hPSC-derived BOs (hPDBOs) provide new opportunities to recapitulate the complicated processes during brain development, neurodegenerative disorders, and brain cancers in vitro. In this review, we will provide an overview of existing BO models and summarize the applications of this technology in modeling the neural disorders and cancers. Furthermore, we will discuss the challenges associated with their use as in vitro models for disease modeling and the potential future direction.
\end{abstract}

Keywords: Human pluripotent stem cells, Brain organoids, Neural disorders, Cancer

\section{Overview of hPDBOs}

The complexity of human brain, including the cell type diversity, cellular architecture and functional connectivity, makes understanding its secrets to be one of the most fascinating things in science [1]. However, the difficulty in accessing of human brain tissues greatly limited our investigation and understanding to various of neurological disorders. Although some studies using the animal model provide valuable mechanistic insights into the pathogenesis and causes of human brain disorders, their values are limited because of the species differences. Therefore, it is urgent to develop an alternative experimental system to study human brain development and disorders, and which should be able to mimic the human context and ethically just.

*Correspondence: lipeng56@mail.sysu.edu.cn Scientific Research Center, The Seventh Affiliated Hospital of Sun Yat-Sen University, Shenzhen 518107, China
hPSCs, including embryonic stem cells (ESCs) and induced pluripotent stem cells (iPSCs), can propagate in vitro and differentiate into all adult cells, they therefore hold great promise for disease remodeling and drug discovery [2-5]. Particularly, the iPSCs derived from patients substantially facilitated us to recapitulate some of the disease-associated phenotypes with brain disorders from the cellular level [6]. Combination of genomeediting technology with hPSC model enables scientists to recapitulate any disease-associated mutations in cultured cells, and further correct of these genetic mutations in patient-derived iPSCs can be used to validate these phenotypes [7-9]. These technologies indeed prove a boon for revealing cellular phenotypes associated with brain development and disorders [10]. However, most of the disease phenotypes are still difficult to be recapitulated using the monolayered culture system, owing to lack of the tissue architecture and microenvironment. To this end, the hPDBOs, which are self-organized in vitro and resemble the embryonic human brain, including cell type

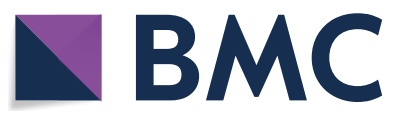

c) The Author(s) 2021. This article is licensed under a Creative Commons Attribution 4.0 International License, which permits use, sharing, adaptation, distribution and reproduction in any medium or format, as long as you give appropriate credit to the original author(s) and the source, provide a link to the Creative Commons licence, and indicate if changes were made. The images or other third party material in this article are included in the article's Creative Commons licence, unless indicated otherwise in a credit line to the material. If material is not included in the article's Creative Commons licence and your intended use is not permitted by statutory regulation or exceeds the permitted use, you will need to obtain permission directly from the copyright holder. To view a copy of this licence, visit http://creativeco mmons.org/licenses/by/4.0/. The Creative Commons Public Domain Dedication waiver (http://creativecommons.org/publicdomain/ zero/1.0/) applies to the data made available in this article, unless otherwise stated in a credit line to the data. 
diversity and cytoarchitecture [11-14], provide a unique platform to investigate the complicated processes during brain development, neurodegenerative disorders and tumorigenesis $[15,16]$.

In 2008, Eiraku et al. firstly adopted a 3D aggregate culture system to differentiate ESCs into cortical progenitors, the resulting embryoid body (EB)-like aggregates exhibited regional dorsal-ventral specification and recapitulated the early aspect of corticogenesis $[17,18]$. In 2013, Lancaster et al. modified this protocol by introducing Matrigel droplets and Spinning bioreactor to maintain 3D structure of the aggregates and continuous supplies of oxygen and nutrients, the resulting aggregates (called BOs) displayed structural features like human brain, such as establishing the apical-basal polarity and expressing region-specific markers within the cortical domain, including forebrain, midbrain, hindbrain, hippocampus etc. $[11,19,20]$. Subsequently, by extending the culture time with an improved Spinning mini-bioreactor or a sliced neocortical organoid (SNO) system, an expanding cortical plate was developed, which constitutes neural subtypes of all six cortical layers and resembles with the third trimester embryonic human neocortex [21, 22]. Transcriptome comparisons of human BOs and fetal neocortex at different stages by scRNA-seq showed the organoids also closely recapitulated the development of glial lineage cells, and their gene expression patterns were reminiscent of fetal human brain development $[12,14,23-26]$. More importantly, when the in vitrodeveloped human BOs were transplanted to adult mouse brain, they showed progressive neural differentiation, maturation, and established the graft-to-host functional synaptic connectivity, which facilitated us to model brain diseases under physiological conditions [27].

Up to now, various types of defined protocols have been developed to differentiate hPSCs to region-specific BOs, including forebrain, midbrain, hindbrain, hippocampus, choroid plexus, hypothalamus, and cerebellum organoids, all of them have recapitulated the molecular, cellular, and structural features of the corresponding areas of human brain [21, 28-32]. Relying on these technologies, the researchers have developed the "assembloids" by fusing differentially patterned region-specific BOs, such as forebrain dorsal and ventral fusion, and forebrain fusion with thalamus [33-38]. These assembloids enable us to explore the complex interactions between different parts of brain, and will shed light on more complicated processes of brain development and diseases. In 2019, Giandomenico et al. developed an air-liquid interface cerebral organoid (ALI-CO) system, and by culturing with mouse spinal cord sections, the resulting organoids have established synapses with spinal cord neurons, which could further guide mouse muscle contraction [39]. Recently,
Andersen et al. have assembled BOs and spinal cord-like organoids, together with human skeletal muscle spheroids to generate the cortico-motor assembloids. In this model system, they observed the corticofugal neurons can project and connect with spinal spheroids to control muscle contraction [40]. These studies highlighted the remarkable self-assembly capacity of 3D cultures to form a functional circuit, and which could be used to understand previously inaccessible features of human brain function, such as cell-cell interactions and neural circuit formation in whole central nervous system (CNS) [41-43].

In the last decade, the hPDBO model, including the whole brain, region-specific BOs and assembloids (Fig. 1 and Table 1), has been widely used to investigate human brain disorders and cancers (Table 2). In this review, we summarize the current applications of hPDPOs in these aspects, and will discuss the challenges of their use as in vitro models and the potential future directions.

\section{BOs for modeling neurodevelopmental disorders}

Neurodevelopmental disorders are the diseases that impair brain functions such as emotions, learning, sociality, or self-control due to perturbations in the developmental processes. Microcephaly, Epilepsy, and Autism spectrum disorder (ASD) are well known examples [44]. More than $5 \%$ of the world population is suffering from neurodevelopmental disorders [45]. However, the pathological causes of them are largely unknown due to lack of a proper preclinical model by which to study the disease mechanisms and develop new treatments. hPDBOs are expected to provide new breakthroughs in this field, owing to the similarities of BOs and human brain in origin and development processes. In this section, we summarize recent findings using hPDBOs for neurodevelopmental disease modeling and drug screening.

\section{Modeling microcephaly with $\mathrm{BOs}$}

Microcephaly is a typical neurodevelopmental disorder characterized by markedly reduced brain size, and the thin and not well layered cortical layers in patients [46]. Microcephaly is classified as congenital and acquired in terms of the cause of this disease, and lots of mouse models have been used to study the pathogenesis of genetic microcephaly. However, they were unable to closely recapitulate the microcephaly-associated phenotypes like in human patients, due to lack of the outer radial glia cells (oRGs) in human brain, which have been identified to be responsible for differentiation of the majority of upperlayer neurons [47-49]. hPDBOs have been demonstrated to contain these specific neural oRGs, and are eminently suitable for microcephaly modeling and drug screening $[12,25,50]$. So far, three independent centrosome-related 


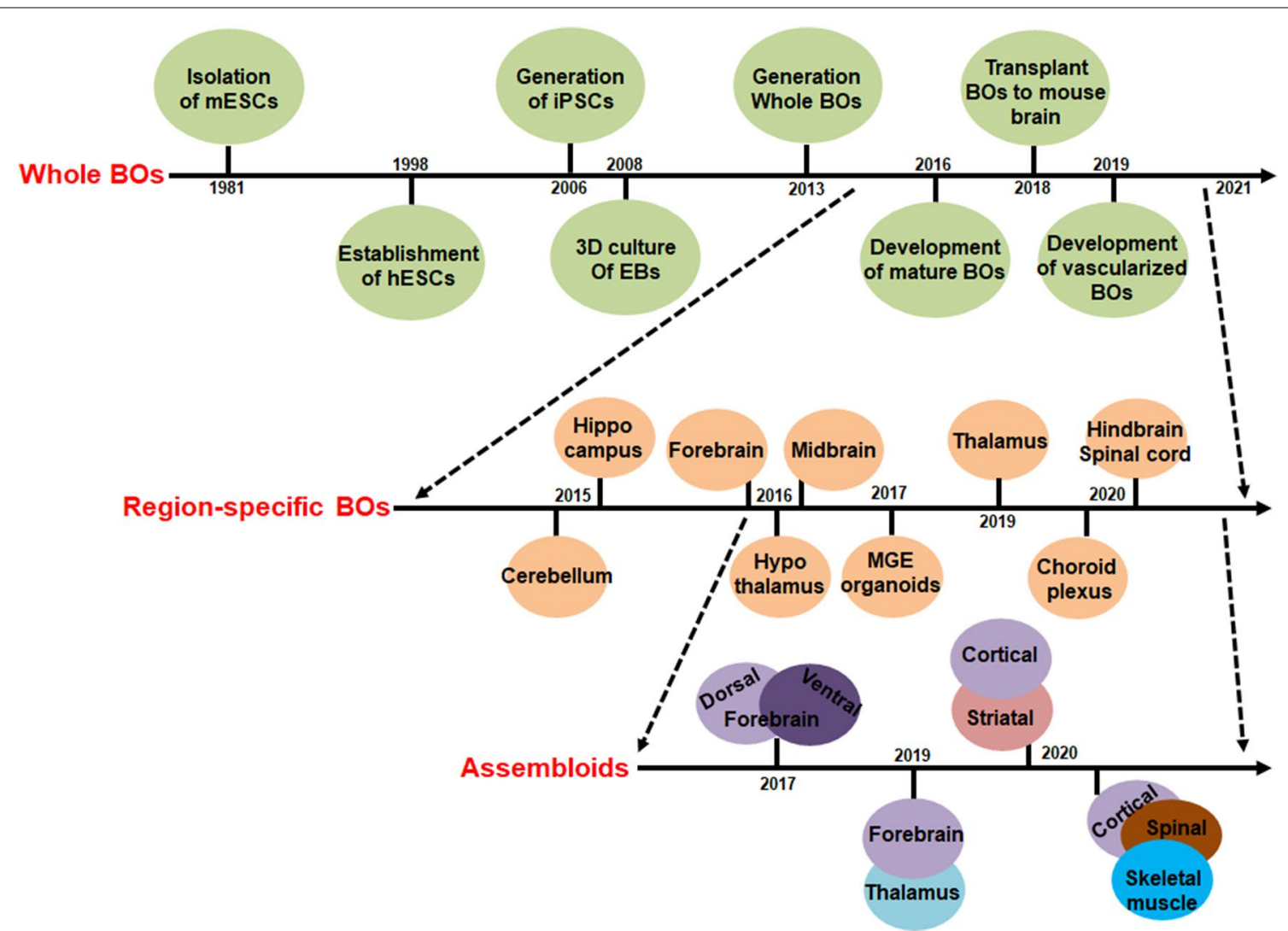

Fig. 1 Development of brain organoid technologies: Timeline cartoons to indicate the milestones from isolation of $\mathrm{m} / \mathrm{hESCs}$ to establishment of mature hPDBOs, including vascularized BOs, region-specific BOs and assembloids

BOs have been generated from microcephaly patient iPSCs carrying mutations in CDK5RAP2, CPAP and $A S P M[11,51,52]$. CDK5RAP2 is a pericentriolar material protein $(\mathrm{PCM})$ in a centrosome and its mutations will lead to aberrant centrosomes function [53-55]. In 2013, Lancaster et al. firstly generated the patient iPSC-derived BOs carrying a compound heterozygous nonsense mutation in CDK5RAP2, and these organoids present a smaller overall size and reduction of the progenitor zones compared to controls, because of the abnormal proliferation and differentiation of oRGs in patient organoids [11]. In addition, as a CDK5RAP2-interacting protein, CPAP is a centriole wall protein required to assemble and recruit PCM proteins to a developing centrosome [56, 57]. Despite several independent CPAP mutations have been identified in microcephaly patients, the underlying mechanism is unknown. In 2016, Gabriel et al. generated stable iPSCs from microcephaly patient-derived fibroblasts with a deletion of exons 11-13 in CPAP. These patient iPSC-derived BOs exhibit a reduced size, enlarged ventricular lumen, increased number of cilia as well as premature neurogenesis, owing that the mutant CPAP protein caused cilia disassembly, and eventually lead to a delayed cell-cycle re-entry and premature neurogenesis [51]. These results suggested that maintaining the normal centrosome function play a critical role in expanding human neural progenitor cells (NPCs) and avoiding of microcephaly. Mutations in WDR62 are the second most common genetic cause of autosomal recessive primary microcephaly in human [58]. Using the WDR62-deficient hPDBOs, Zhang et al. showed that WDR62 depletion resulted in smaller BO sizes due to reducing the oRGs proliferation and inducing the early neurogenesis. On a molecular level they identified a novel WDR62-CEP170KIF2A pathway as a contributor to microcephaly [59]. These studies demonstrated the hPDBOs provide a reliable platform to recapitulate the microcephaly-associated phenotypes and identify the molecular causes of microcephaly. Additionally, this model can be used to screen new microcephaly-associated genes. Wang et al. identified biallelic missense and frameshift mutations in NARS1 in seven patients from three unrelated families with microcephaly, and these patient-specific BOs showed reduced proliferation of RGCs, resulting in smaller organoid characteristics of microcephaly, suggesting that NARS1 is required to support RGC 


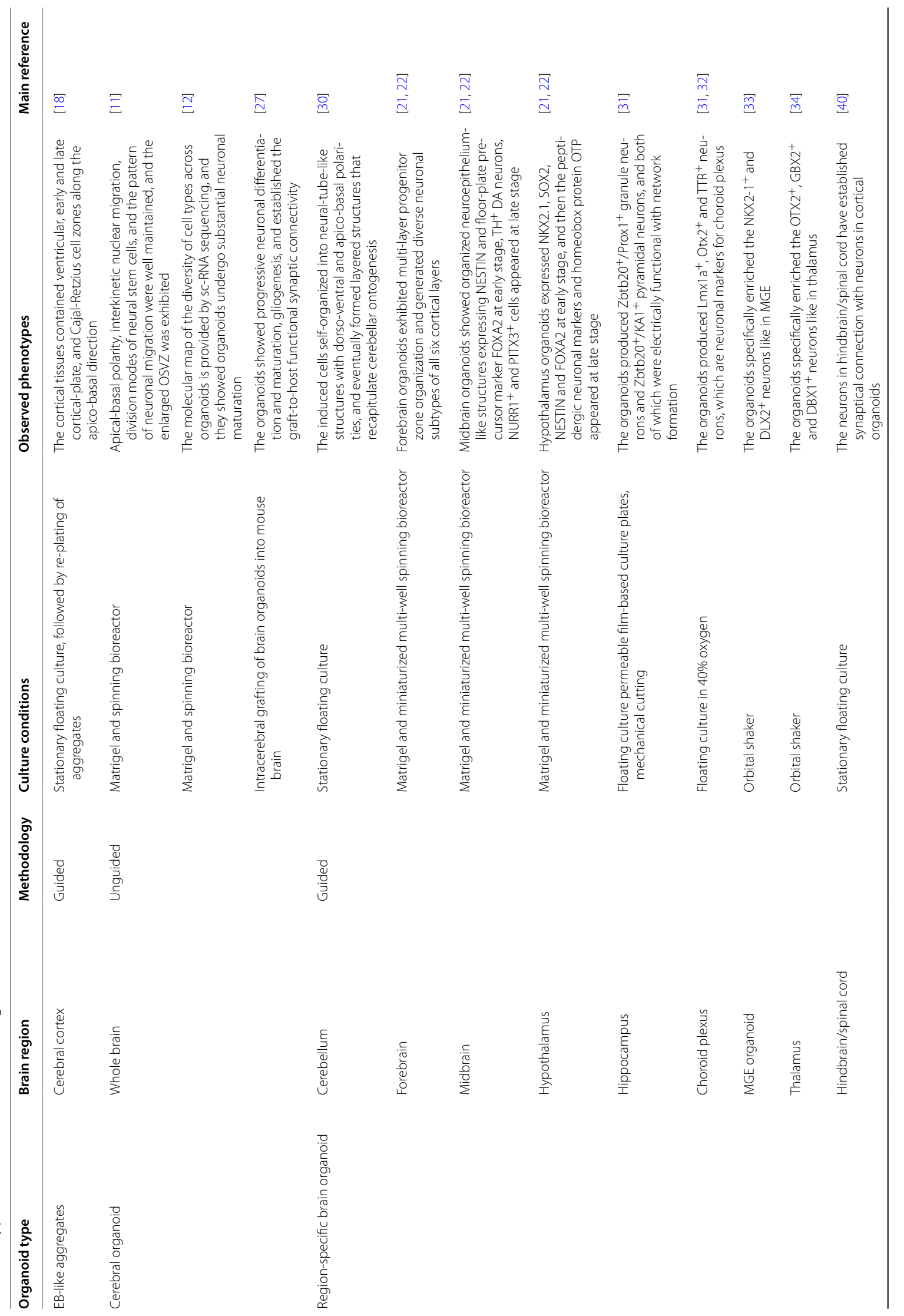




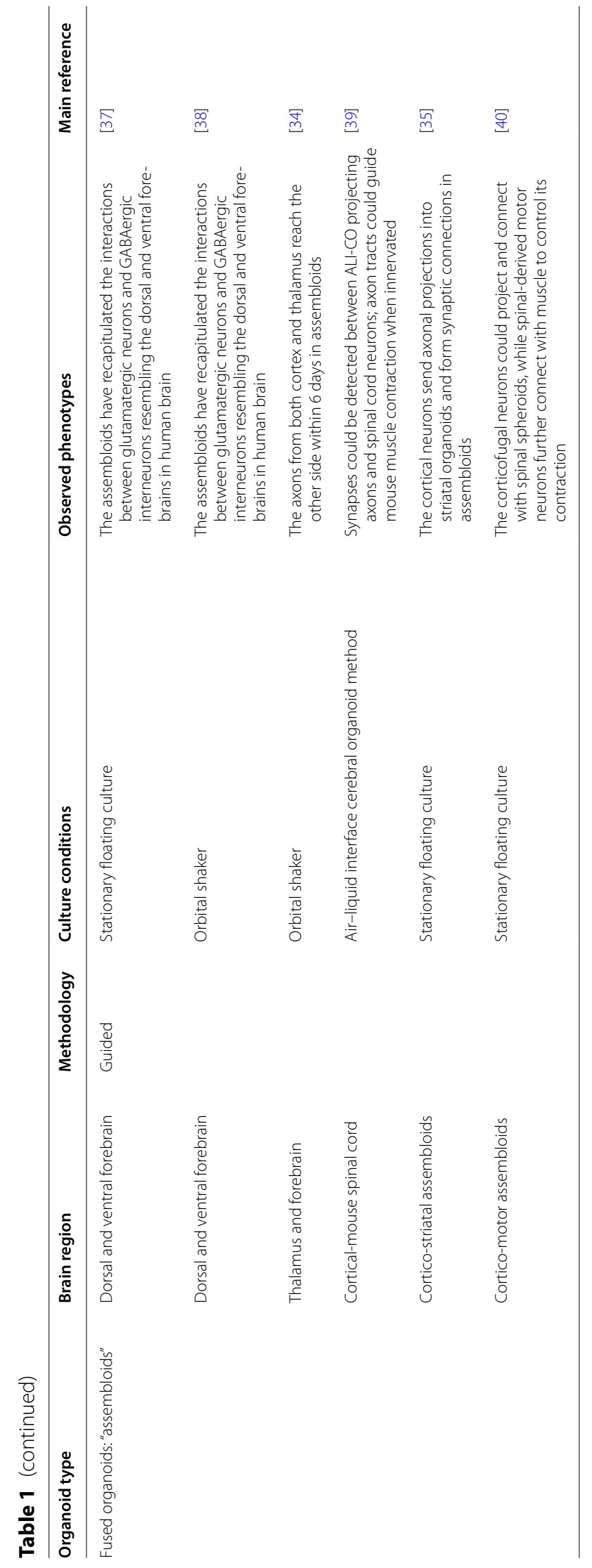




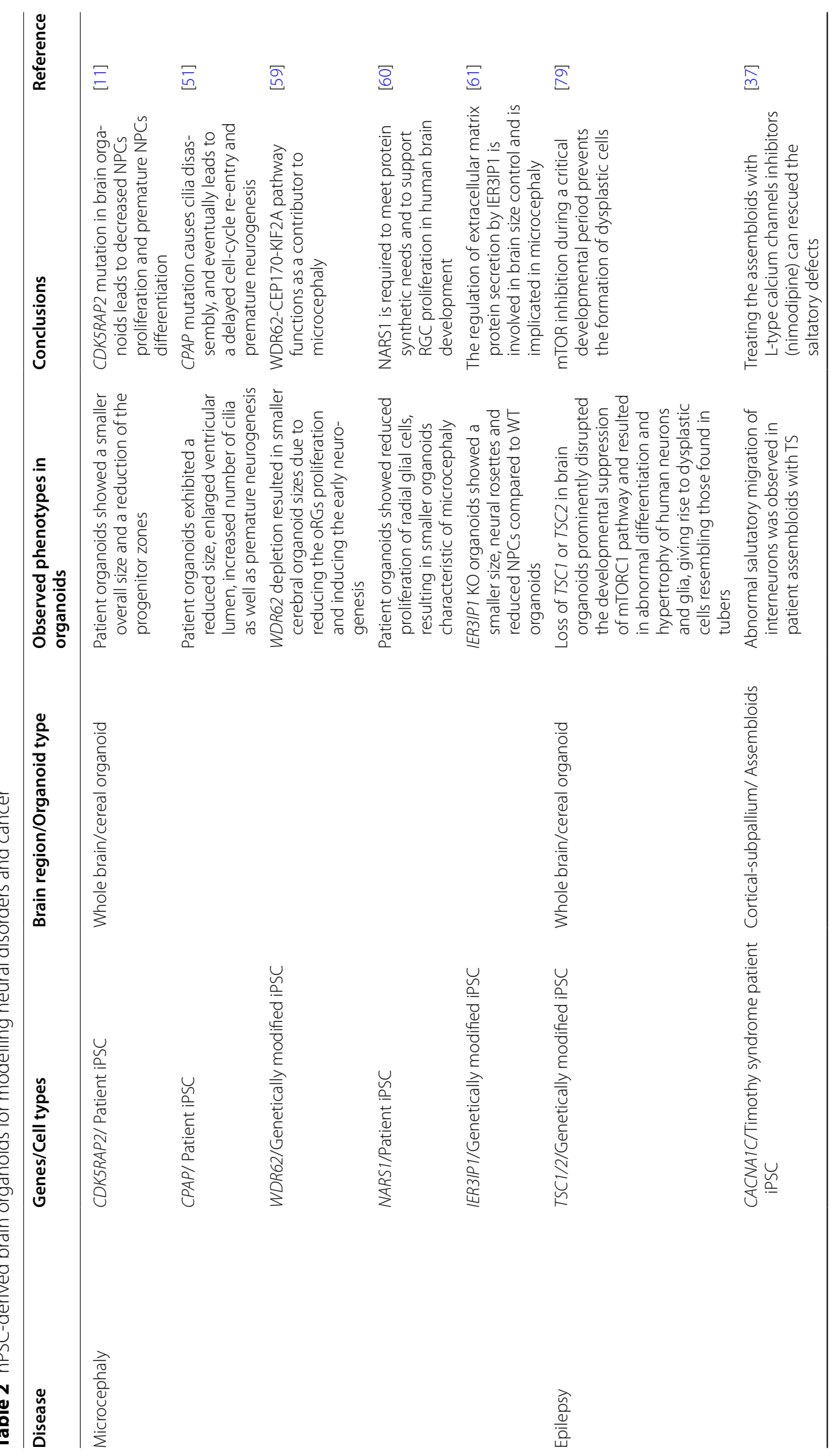




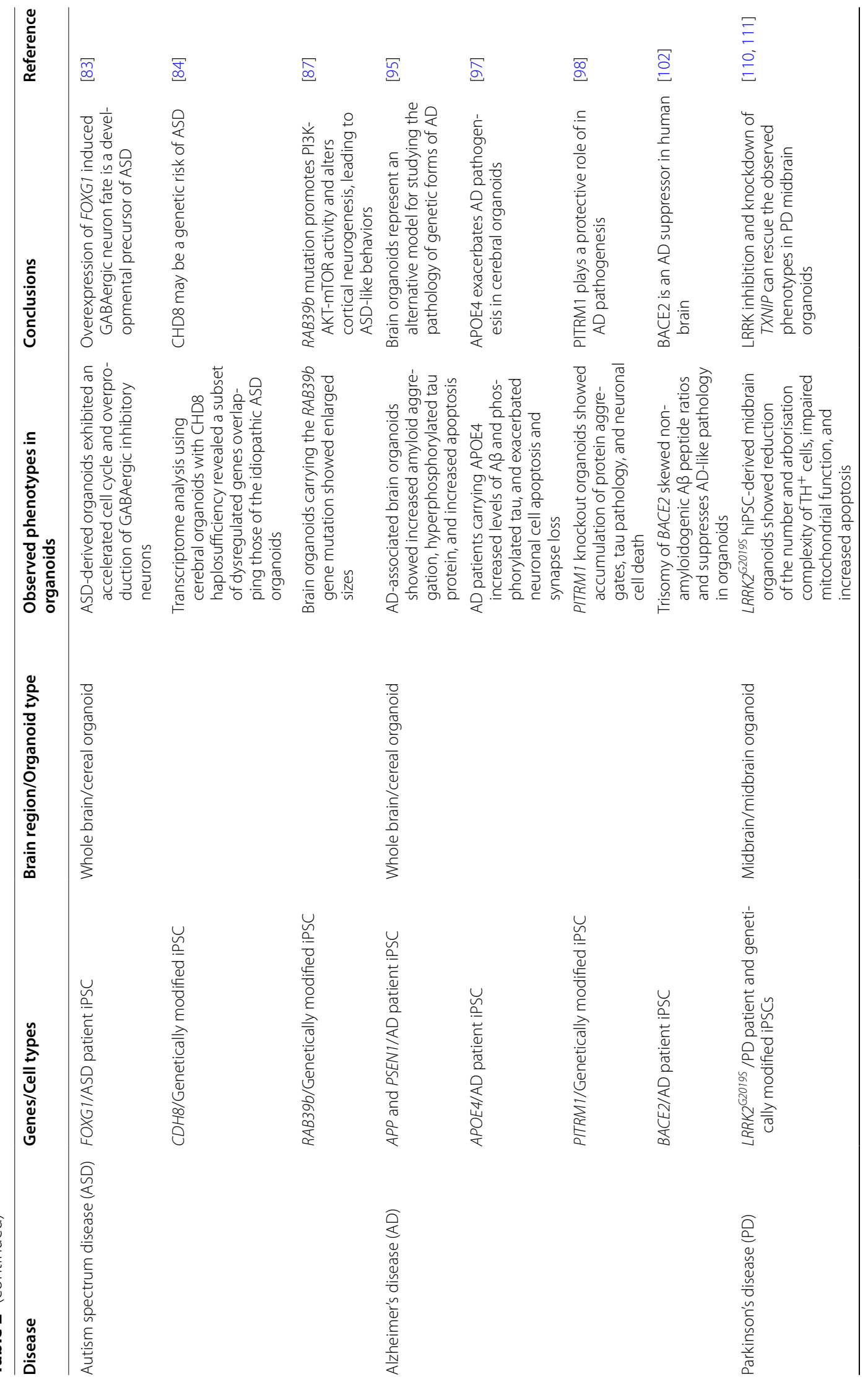




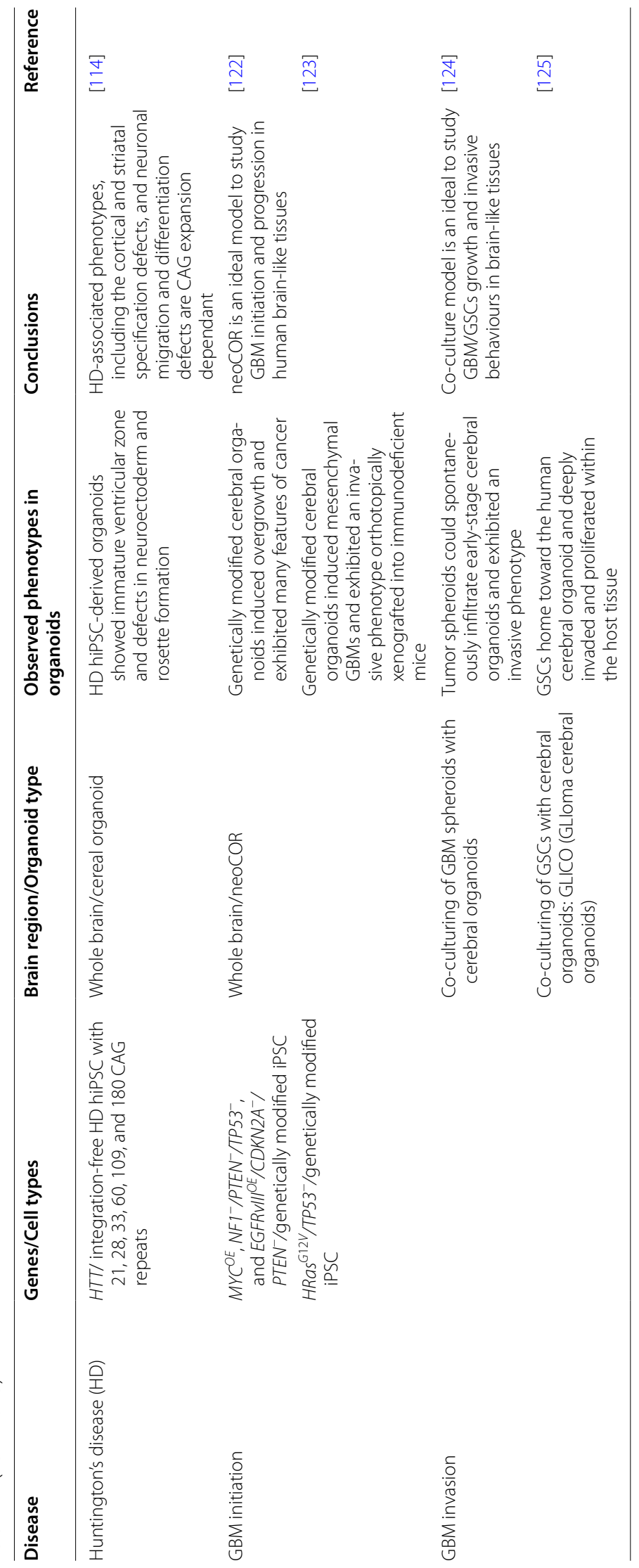


proliferation in human brain development [60]. Recently, Esk et al. performed a loss-of-function (LOF) screen by CRISPR-LIneage tracing at cellular resolution in human BO tissue, and they tested 173 microcephaly candidate genes and identified 25 of them to be involved in known and uncharacterized microcephaly-associated pathways [61]. In summary, hPDBOs facilitated us to identify new microcephaly genes, and mechanisms involved in brain size control and drug screen in the future.

Besides the inherited microcephaly, Zika virus (ZIKV) pandemic in the Americas has demonstrated to be able to cause microcephaly [21, 62-64]. BOs exposed to ZIKV at different developmental stages showed ZIKV could directly target NPCs at the ventricular zones and cause depletion of NPCs, leading to the overall size reduction of organoids as seen with genetically inherited primary microcephaly $[65,66]$. Subsequently, using the human forebrain organoid model, they further revealed that ZIKA-NS2A reduced RGC proliferation by impairing adherens junction complex formation and aberrant radial glial fiber scaffolding [67]. Moreover, several drug screens followed with validation using $\mathrm{BO}$ model have identified compounds such as emricasan, hippeastrine, 25-hydroxycholesterol and certain antibiotics and antivirals as promising candidates for treating Zika virus infection [68-71].

\section{Modeling epilepsy with BOs}

Epilepsy is one of the most common neurological disorders and characterized by recurrent and unprovoked seizures due to neuronal hyperactivity. Epilepsy is etiologically heterogenous and antiepileptic drugs are the primary treating method. However, approximately $40 \%$ of patients have medically refractory seizures [72, 73]. So far, more than 500 loci of gene mutations are identified to be associated with epilepsy, but the molecular mechanisms remain largely unknown, even though they ultimately lead to brain hyperexcitability [74]. For genetic epilepsy, understanding the pathological mechanism is critical in identifying innovative therapeutic approaches. hPDBOs, with its more complex spatial structure, cell diversity and mature neural networks, have been widely used for modeling epilepsy-related disorders. Tuberous sclerosis complex (TSC) is an autosomal dominant congenital disorder characterized by growing benign tumors in multiple organs $[75,76]$. Tumors presenting in brain, accumulating lots of enlarged and dysplastic neurons and glias in the cortex, are associated with epilepsy [77]. The LOF mutations of either TSC1 or TSC2 genes, which encode hamartin and tuberin respectively, will lead to the cortical tubers in brain due to hyperactivation of mTOR pathway that causes aberrant cell growth [78]. Blair et al. have generated hPDBOs carrying the null allele of TSC1 or TSC2 by gene-editing technology, and found homozygous loss of TSC1 or TSC2 exhibited the phenotypes resembling those found in tubers [79]. Timothy syndrome (TS) is another neurodevelopmental disorder characterized by epilepsy [80]. Birey et al. developed an in vitro assembloid by fusing human cortical spheroid (hCS) and subpallium spheroid (hSS) derived from TS patients. These hCS-hSS organoids showed an increase in saltation frequency, as well as a decrease in saltation length and speed, and which could be rescued by treating the assembloids with L-type calcium channels inhibitors, suggesting LTCC blockers might be used to prevent migration defects in patients with TS [37]. Undoubtedly, these studies demonstrated that hPDBOs provided a reliable platform for genetic epilepsy modeling and drug screening.

\section{Modeling ASD with BOs}

ASD is a neurodevelopmental disorder and characterized by the defects of social interaction and communication, as well as repetitive behaviors. ASD is involved in the cell-cell interactions of many different cell types in brain, which makes it difficult to model with traditional 2D cell culture. Its pathophysiology remains elusive [81, 82]. The emergence of hPDBOs, in particular the assebmloids, holds this potential, as they can establish discrete regions of the brain [12, 14, 23, 24]. So far, some of the ASD-associated mechanisms have been identified using these models. In 2015, Mariani et al. generated BOs using the patient iPSCs with idiopathic ASD and demonstrated these organoids exhibit an accelerated cell cycle and overproduction of GABAergic inhibitory neurons, due to the overexpression of transcription factor FOXG1, revealing that FOXG1-induced GABAergic neuron fate is a developmental precursor of ASD [83]. CHD8, one of the most frequently mutated genes in ASD, encodes a member of ATP-dependent chromatin-remodeling factors [84]. The iPSCs carrying the $\mathrm{CHD} 8^{+/-}$mutation have been used to generate the $\mathrm{BOs}$ and subjected to do RNA-sequencing analyses. The results showed that $\mathrm{CDH} 8$ regulates the expression of genes implicated ASD, such as TCF4 and AUTS2. In particular, the differentially expressed genes (DEGs) identified in this study showed extensive overlap with the DEGs found in the Mariani study, including the GABAergic interneuron development-related genes [85]. These studies suggested that abnormal development of GABAergic neuron is one of the common pathological mechanisms of ASD. RAB39b is an X-linked gene and codes for a member of the RAS-like GTPase superfamily. LOF mutations of $R A B 39 b$ lead to ASD [86]. hPDBOs carrying the $R A B 39 b$ mutation showed enlarged sizes due to the overproliferation and impaired differentiation of NPCs, further studies revealed $R A B 39 b$ mutation 
promotes PI3K-AKT-mTOR activity and alters cortical neurogenesis, leading to macrocephaly and autistic-like behaviors [87]. Recently, hPSC-based thalamic organoids and cortex-thalamus assembloids have been developed in vitro, which may hold great promises to do ASD modeling and drug screening in depth [35].

\section{BOs for modeling neurodegenerative disorders}

Neurodegenerative diseases affect millions of people worldwide. Parkinson's disease (PD), Alzheimer's disease (AD), and Huntington's disease (HD) are typical neurodegenerative diseases caused by progressive death of neurons in brain. Despite the neurodegenerative diseases have been widely studied using various of animal models, they failed to fully recapitulate all the disease features in human [88-90]. In this section, we summarize recent findings using hPDBOs for neurodegenerative disease modeling and drug screening.

\section{Modeling AD with BOs}

$\mathrm{AD}$ is an age-related neurodegenerative disease, and clinically manifestated with progressive memory loss and cognitive dysfunction [91]. The main pathological features of $A D$ patients were accumulation of amyloid $\beta$-peptide $(A \beta)$ peptides and hyperphosphorylated Tau in brain, which will induce the formation of senile plaques and neurofibrillary tangles, and eventually the neuronal cell loss [92]. Previous studies have repetitively shown the existing models, including the monolayer cell culture system and transgenic animal models, were unable to fully recapitulate all of the disease features, due to lack of brain tissue organization and existing of species differences [93]. Even more, the drugs developed from the AD mouse models have failed to demonstrate efficacy in human patients [89]. Thus, developing a more effective and predictable model is a top priority for AD modeling and treatment. hPDBOs, either from AD patient or carrying the $A D$ patient-related mutations, have spontaneously developed the AD pathological features, including accumulation of $A \beta$ aggregates and hyperphosphorylated Tau [94-96]. In 2018, Gonzalez et al. firstly showed familial AD patient iPSC-derived BOs developed the two major pathological features of $\mathrm{AD}$ and demonstrated the $\mathrm{BOs}$ may represent an alternative model for studying the pathology of genetic forms of AD [95]. Subsequently, Zhao et al. showed BOs from AD patients carrying $A P O E 4$ increased levels of $\mathrm{A} \beta$ and phosphorylated Tau, and exacerbated neuronal cell apoptosis and synapse loss, confirming that APOE4-mediated degenerative pathways contributing to AD pathogenesis [97]. Additionally, the $\mathrm{BO}$ model also provided a platform for identification of new AD-risk genes. José Pérez et al. firstly revealed that PITRM1 knockout iPSC-derived BOs spontaneously developed pathological features of $\mathrm{AD}$ and revealed PITRM1 played as a protective role in AD pathogenesis [98]. BACE2 is a homologue of BACE1, however, its expression and function in brain is still confusing [99-101]. Using the hPDBO model, Alić et al. have proved that BACE2 is a dose-sensitive ADsuppressor gene in human brain [102]. Most recently, Park et al. have developed a network-based drugscreening platform by integrating mathematical modeling and hPDBOs with the AD pathological features. Using this platform, they have identified several FDAapproved drugs as candidates for curing AD [103]. Taken together, these studies have demonstrated that hPDBOs indeed represent a more ideal and efficient model for $\mathrm{AD}$ modeling and drug screening.

\section{Modeling PD with BOs}

$\mathrm{PD}$ is the second most frequent neurodegenerative disorder after $\mathrm{AD}$, and characterized by its motor symptoms and the frequent co-occurrence of cognitive and psychiatric symptoms. The prominent pathological changes of $\mathrm{PD}$ are degeneration and death of dopaminergic neurons (DANs) in substantia nigra of human midbrain [104]. Due to lack of representative experimental models that can recapitulate the complex multifactorial neurological diseases, the progress in understanding the molecular mechanisms of PD and discovering the disease-modifying treatments is slow. hPDBO models, in particular the midbrain-specific BOs (MOs), offer new possibilities to overcome this issue [21, 105-108]. In 2016, Qian et al. developed the MOs from hPSC using a miniaturized spinning bioreactor, which recapitulated the key dynamic features of developing human midbrain at the molecular, cellular, and structural level. For example, the MOs contained the ventricular zone (OTX2/FOXA2 ${ }^{+}$), as well as intermediate (LMX1A/NURR1 $\left.1^{+}\right)$and mantle layers $\left(\mathrm{MAP} 2 / \mathrm{TH}^{+}\right)$neurons $[21,29]$. In addition, several pan-mDA neural markers, including the dopamine transporter and DOPA decarboxylase enzyme have been consistently observed in hMOs [108]. Using this model, LRRK $2^{\mathrm{G} 2019 S}$-associated hMOs have been developed [109], and which have recapitulated the PD-relevant phenotypes, including reduced the numbers of mature DANs and neurite length in comparison to the control organoids. Furthermore, treatment of the LRRK2 ${ }^{G 2019 S}$ mutant organoids with LRRK2 inhibitor partially rescued the DANs-specific gene expression $[110,111]$. These studies showed hMOs may represent an efficient and reliable platform for PD modeling and drug screening in the future. 


\section{Modeling HD with BOs}

$\mathrm{HD}$ is an autosomal dominant neurodegenerative disease and characterized by motor dysfunction, progressive cognitive deterioration and the behavioral prodrome [112]. it is caused by abnormal expansion of CAG repeat in Huntingtin gene $(H T T)$, more than 40 of CAG repeats will confer HD and induce the neural cell death in the striatum and cortex [113]. Previous study has demonstrated BOs-derived from the HD iPSCs with increasing CAG expansions caused the failure of neuroectodermal acquisition, disruption of neural rosette and organoid cytoarchitecture, which could be rescued by knockdown of HTT or pharmacologic inhibition of ADAM10 activity, indicating these phenotypes are dependent on CAGexpansion [114]. However, whether the BO models used here correctly mimic what happens in HD patients still need to be investigated. Recently, Andersen et al. have developed a three-part assembloids, consisting of BOs, spinal cord and skeletal muscle spheroids, which can be maintained functionally and morphologically intact for up to 10 weeks in vitro, and may represent a resource for uncovering mechanistic insights into HD and investigating new HD treatment strategies [40].

\section{BOs for modeling tumor initiation, progression, and invasion}

The complexity and heterogeneity of cancers, lead to the variations in the curative effects from person to person, which is an enormous challenge faced in tumor therapy. Developing an experimental model that accurately recapitulates cancer is crucial for the study of cancer biology and development of therapeutic treatments. Traditional in vitro culture models, including 2D human cancer cell or 3D tumor spheroid cultures, are most likely to acquire additional mutations during amplification process due to the genomic instability; while transgenic animal models cannot fully reflect the genetic characteristics of human cancers; patient-derived xenotransplantation models can retain the important features of the primary tumors, however, they are not suitable for high-throughput drug screening owing to the high cost and low implantation efficiency. hPSC-derived 3D organoids are easily accessible and handled, and have more closely recapitulated the relative complicated cytoarchitecture and microenvironment of human organs. Therefore, many of them have been generated to study tumorigenesis and personalized therapy, including glioblastoma (GBM), pancreatic ductal adenocarcinoma (PDAC), lung adenocarcinomas, retinoblastoma, and colorectal cancer organoids [115-120]. Using this model, the researchers have uncovered new mechanistic insights into the tumor development, which are difficult to be learned from other models. For example, PDACs can develop from both acini and ducts, and acinar cell-derived PDACs frequently traverse to pancreatic intraepithelial neoplasia, while PDACs developed from ductal cells are more likely to progress to aggressive cancers [121, 122]. However, how does the cell type of origin affect human PDAC biology and progression is unknown. For this issue, one of the challenges is human acinar cell cultures are short lived and easy to undergo trans-differentiation by acinar-to-ductal metaplasia, while the mouse model is not in a human cell context. Recently, two separate groups from Huang et al. and Breunig et al. have generated the hPSC-based pancreatic duct-like and acinus-like organoids, which have recapitulated the properties of neonatal exocrine pancreas. Using these models introducing the PDAC-associated oncogene mutations, they revealed that $G N A S^{R 201 C}$ and $K R A S^{G 12 D}$ have lineage-dependent effects on PDAC formation in vitro and in vivo, GNAS ${ }^{R 201 C}$ mutated in ductal organoids induced cystic growth more effectively than acinar organoids, whereas $K R A S^{G 12 D}$ expressed in acinar organoids is more effective to induce acinar-to-ductal metaplasia-like changes and model PDAC in vivo $[119,120]$. This study highlighted the advantages of hPSC-derived organoids in modeling tumor progression of heterogeneity. GBM is the most common intracranial tumor with rapid growth, strong invasion, and easy recurrence. The prognosis of GBM with the highest malignant degree is extremely poor, and the median survival time is less than 15 months [123]. Currently, the main treatment strategies for GBM are by surgery combined with postoperative radiotherapy and chemotherapy. Targeted treatment and immunotherapy strategies are very limited, owing to the presence of blood-brain barrier (BBB) and lack of knowledge mechanism underlying the tumor initiation and progression $[124,125]$. Although many models have been developed for preclinical modeling of GBM, their contributions to clinical treatment are very limited due to its molecular heterogeneity and complicated cytoarchitecture and microenvironment of human brain tissue [126, 127]. In this part, we will summarize the recent applications of BOs during the study of GBM initiation, progression, and invasion.

\section{BOs for modeling GBM initiation and progression}

In the last 3 years, hPDBOs have been widely used to model the initiation and progression of GBM $[128,129]$. For example, in 2018 , by combining with genome-editing technology, hPDBOs have been successfully induced to grow GBM in vitro, which is also called neoplastic cerebral organoids (neoCORs). Using this model, Bian et al. identified that overexpression of oncogene $M Y C$ or introducing other commonly found gene aberrations from patient GBM into wild type BOs, can strikingly induce tumor overgrowth, which have exhibited many features 
of cancer, like in vivo expansion and invasion capabilities [116]. More interestingly, they found the induced neoCORs by overexpressing $M Y C$ showed histopathological features, cellular identities, and transcriptome signatures very similar to those described for human central nervous system primitive neuroectodermal tumor (CNSPNET), for which no successful animal or in vitro model exists so far [130]. More importantly, $M Y C^{O E}$ alone could initiate CNS-PNET-like neoplasm in BOs within a very short period, while normally it requires additional genetic events such as loss of $p 53$ and much longer time in animal models, with low incidence [131]. Using the similar strategy, Ogawa et al. have shown activation of oncogene HRas ${ }^{G 12 V}$ and simultaneous disruption of TP53 can induce mesenchymal-like GBM in organoids, and the cells isolated from these organoids can be orthotopically xenografted into immunodeficient mice and exhibited an invasive phenotype. Transcriptome analysis of these organoid-generated putative tumor cells showed gene expression profiles consistent with mesenchymal subtype human GBM [132]. Taken together, these studies demonstrated that neoCORs can be used to model the initiation of different types of GBMs with specific DNA aberrations, including the highly rare and malignant tumors.

\section{BOs for modeling GBM invasion}

GBM is more prone to propagate and ultimately kill patients through diffuse invasion and infiltration into surrounding normal cerebral tissue, instead of by metastasis to other tissues. Thus, 2D cultures and traditional 3D tumor organoids cannot model this critically important cell-cell interactions and the tumor microenvironment, while the other animal models exist marked interspecies differences. hPDBOs cocultured with GBM spheroid or patient-derived glioblastoma stem cells (GSCs) can form glioma BOs to study tumor invasion. Da silva et al. firstly demonstrated that GBM spheroids can spontaneously infiltrate early-stage BOs to form hybrid organoids, which provides a basis for modeling and quantification of the GBM infiltration process [133]. Subsequently, Linkous et al. developed a cocultured system with patientderived GSCs and hPDBOs, and called their model as GLICO (GLIoma cerebral organoids). They showed the GSCs can deeply invade and proliferate in human BOs, which has phenocopied the patient GBM behaviors and provided a system for modeling tumor cell invasion in a human brain environment [134, 135]. More importantly, using this GLICO model, they demonstrated that GSCs grown within the microenvironment of cerebral organoids were more resistant to drug and radiation-induced genotoxic stress, as seen in GBM patient treatment, such an effect is not recapitulated using standard 2D culture system. These studies demonstrated that GLICO provided a powerful tool for investigating GBM biology in a primitive human brain environment and modeling diverse therapeutic interventions.

\section{Challenges for existing BO models}

In the past decade, numerous of breakthroughs have been made in $\mathrm{BO}$ technologies, including the organoid development methodologies and their applications in disease modeling and drug screening. However, this field is still in its infancy, and many of the challenges are existed (Fig. 2).

\section{Limited reproducibility}

One of the major limitations of BOs is their variability within and between organoid batches, which resulted in limited reproducibility and sometimes the data misinterpretation for disease modeling. For example, even in the same batch, BOs generated by unguided protocols showed lots of variations, including the morphologies, sizes and cytoarchitectures $[11,19]$, resulted from inconsistent neural induction efficiency and matrigel supports. To overcome this issue, Lancaster et al. have used poly (lactide-co-glycolide) copolymer fiber microfilaments as a floating scaffold to generate elongated EBs, by which the resulting organoids displayed improved reproducibility of neural induction and subsequent cortical development [136]. In addition, compared with above-mentioned unguided protocols, cortical organoid generated by guided protocols can achieve reproducible generation with a rich diversity of cell types appropriate for the human cerebral cortex $[14,137]$. Recently, Zhu et al. have developed a simple protocol to enable in situ formation of massive BOs from iPSCs on a micropillar array without tedious manual procedures. The optimized micropillar configurations allow for controlled EB formation, neural induction and differentiation, and generation of functional human $\mathrm{BOs}$ in $3 \mathrm{D}$ culture on a single device. By combining microfabrication techniques with stem cells and developmental biology principles, the proposed method can greatly simplify BO formation protocols and overcome the potential limitations of cell contamination, lower throughput and variance of organoid morphology [138]. Overall, during organoid generation, uniform size, uniform morphology, and synchronized differentiation of EBs should be considered in the future for improve the reproducibility.

\section{Lack of a functional vascular system}

The majority of BOs generated using current protocols resemble an early stage of fetal brain [19, 20, 25, 26, 139], and which are not suitable to investigate the physiological and pathological aspects of adult brain, like the late-onset neurodegenerative diseases. The major reason is the BOs 


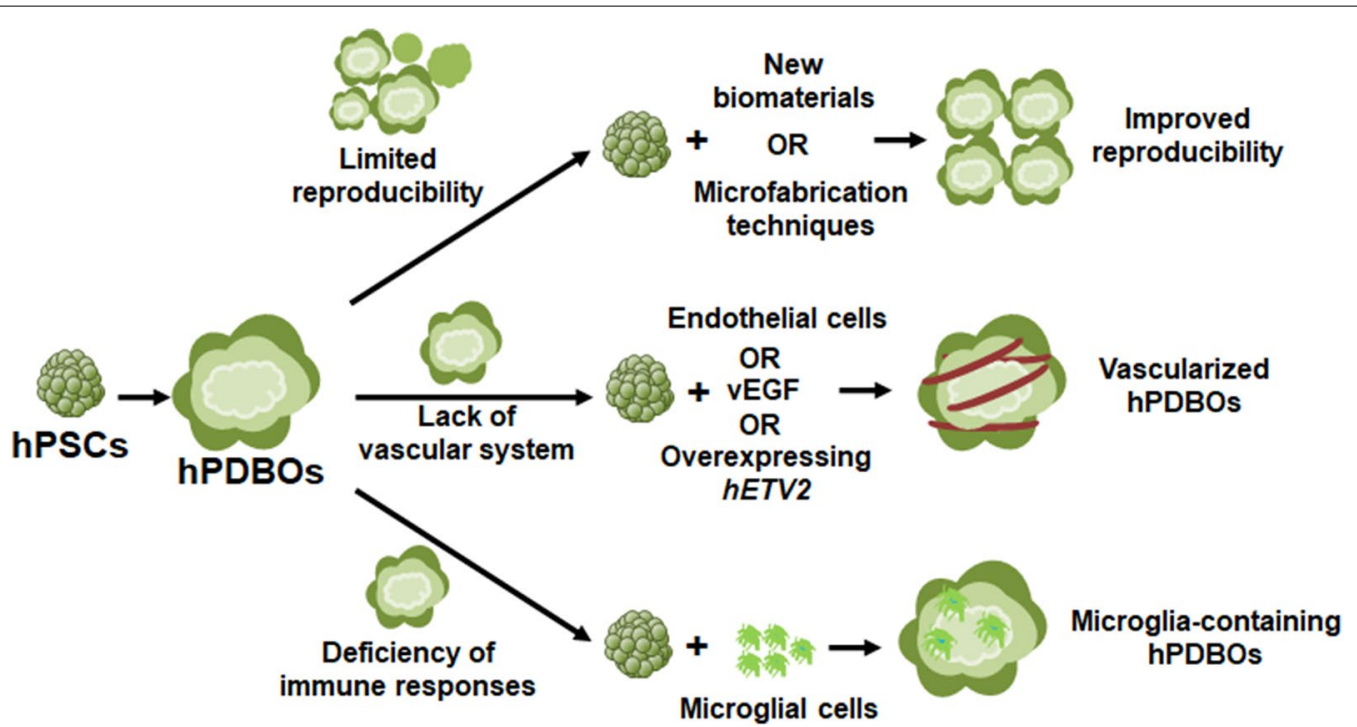

Fig. 2 Challenges of existing hPDBOs: 1. Limited reproducibility: attempt to develop homogenous hPDBOs by introducing new biomaterials or combining with microfabrication techniques; 2 . Lack of vascular system: different attempts to realize organoid vascularization, including hPDBOs co-culture with endothelial cells, or co-differentiation with VEGF administration or co-overexpression with hETV2; 3. Deficiency of immune responses: attempt to develop microglia-containing brain organoids by co-culture of hPDBOs with microglial cells

lack of a circulation system with blood vessels to continually supply of oxygen and nutrients for extended in vitro culture. To overcome this issue, several strategies have been explored to realize the $\mathrm{BO}$ vascularization. BOs co-cultured with endothelial cells or co-differentiated with endothelial-like cells (supplementation of VEGF or overexpression of $h E T V 2$ ) have induced the formation of vascular-like system without reducing neural morphogenesis [140-142]. More recently, Shi et al. have generated the vascularized $\mathrm{BO}$ model (vOrganoids) consisting of typical human cortical cell types and a vascular structure for over 200 days, scRNA-seq analysis illustrated that DEGs of vOrganoids are related to blood vessel morphogenesis. Transplantation of vOrganoids into mouse cortex resulted in the construction of functional humanmouse blood vessels that promoted cell survival in the grafts [143]. In addition, some models have incorporated primary astrocytes, hiPSC-derived pericytes with endothelial cells, astrocytes, and neurons to model the BBB. These BBB models show in vivo characteristics with high transendothelial electrical resistance and expression of tight junction proteins [144-147]. Despite these advances, the BBB platform and differentiation protocols still require optimization to develop functional vasculature in BOs.

\section{Deficiency of immune responses}

Human brain is composed of various types of cells, including neural cells and non-neural cells, such as microglia. Among of them, neural cells are derived from neuroectoderm, while the other cells, such as microglia is from mesoderm. However, most of the current protocols were used to induce the neuroectodermal fate, and the other lineage cells are largely missing in hPDBOs, which greatly limited its application for modeling brain disorders caused by the interactions between non-neural and neural cells. Microglia, as the resident immune cells of CNS, plays essential roles in the initiation and progression of neurodegeneration diseases and cancers [148-150], therefore, microglia induction in BOs would be valuable to study its functions in these diseases. Ormel et al. have reported that BOs generated without dual-SMAD inhibition innately contain mesodermal progenitors, which can differentiate into mature microglia $\left(\mathrm{Iba1}^{+}\right)$under the CNS microenvironment [151]. In addition, co-culturing BOs with hPSC-differentiated microglia-like cells showed a certain degree of response to pro-inflammatory stimuli $[152,153]$. However, creating an environment to induce microglia in BOs that could exhibit inflammation and immune responses like in human brain remains challenging.

\section{Conclusion and future perspectives}

As the only in vitro platform that can recreate the 3D architecture of human brain and recapitulate the process of human neurodevelopment, hPDBOs have made a tremendous breakthrough for modeling brain disorders and cancers. However, it is still with less than a decade 


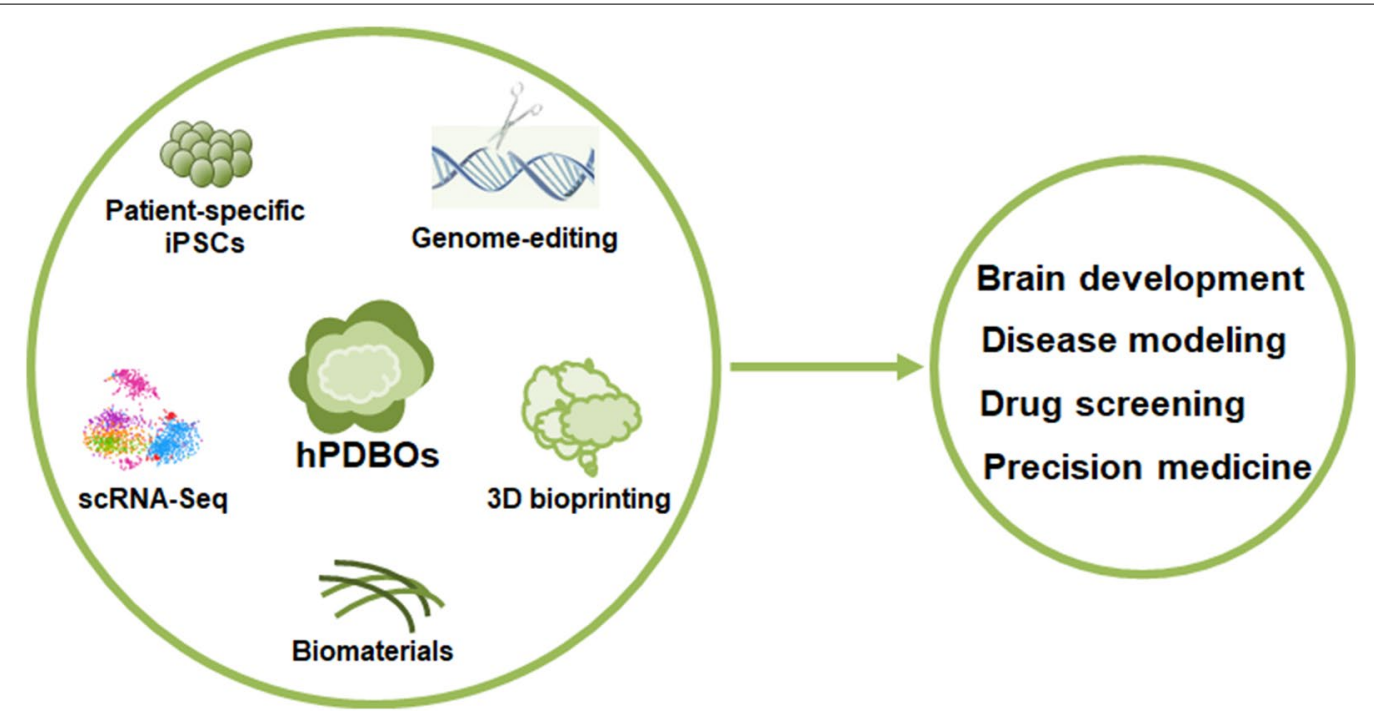

Fig. 3 Future perspectives in hPDBOs: The combinations of hPDBOs and innovative technologies, including cell reprogramming, genome-editing, 3D bioprinting, scRNA-seq, and biomaterials will greatly improve the brain organoid system, and which will facilitate us to model human brain development and disorders, and to perform drug discovery and personalized therapeutics in the future

of history and in its infancy. Continuous advances of 3D-culture systems and their integrations with innovative technologies, including cell reprogramming, genomeediting, 3D bioprinting, single-cell transcriptomics, and biomaterials (Fig. 3), will lead the BOs to become invaluable models for better understanding of the fundamental biology of brain disorders and cancers in the future.

\section{Abbreviations}

3D: Three-dimensional; BOs: Brain organoids; hPSCs: Human pluripotent stem cells; hPDBOs: HPSC-derived BOs; hESCs: Human embryonic stem cells; iPSCs: Induced pluripotent stem cells; EB: Embryoid body; SNO: Sliced neocortical organoid; ALI-CO: Air-liquid interface cerebral organoid; scRNA-seq: Single cell RNA-sequencing; ASD: Autism spectrum disorder; NPCs: Neural progenitor cells; ORGs: Outer radial glia cells; PCM: Pericentriolar material protein; ZIKV: Zika virus; TSC: Tuberous sclerosis complex; TS: Timothy syndrome; hCS: Human cortical spheroids; hSS: Human subpallium organoids; DEGs: Differentially expressed genes; PD: Parkinson's disease; AD: Alzheimer's disease; HD: Huntington's disease; A $\beta$ : Amyloid $\beta$-peptide; DANs: Dopaminergic neurons; MOs: Midbrain-specific brain organoids; HTT: Huntingtin gene; GBM: Glioblastoma; PDAC: Pancreatic ductal adenocarcinoma; neoCOR: GBM organoid neoplastic cerebral organoid; CNS-PNET: Central nervous system primitive neuroectodermal tumor; GSC: Glioblastoma stem cells; vOrganoid: Vascularized brain organoid model; GLICO: GLloma cerebral organoids; BBB: Blood-brain barrier; CNS: Central nervous system.

\section{Acknowledgements}

Not applicable.

\section{Authors' contributions}

$J L$ and $P L$ were responsible for the concept of the review and writing the manuscript. All authors read and approved the final manuscript.

\section{Funding}

This work was supported by the Fundamental Research Funds for the Central Universities (Grant No. 20ykzd02), Guangdong Basic and Applied Basic Research Foundation (Grant No. 2019A1515110285) and National Natural Science Foundation of China (Grant No. 32000679 and 82072901).
Availability of data and materials

Not applicable.

\section{Declarations}

Ethics approval and consent to participate

Not applicable.

Consent for publication

Not applicable.

Competing interests

The authors declare no conflicts of interest.

Received: 7 April 2021 Accepted: 22 May 2021

Published online: 28 May 2021

References

1. Stiles J, Jernigan TL. The basics of brain development. Neuropsychol Rev. 2010;20:327-48.

2. Karagiannis $P$, et al. Induced pluripotent stem cells and their use in human models of disease and development. Physiol Rev. 2019;99:79-114.

3. Thomson JA, et al. Embryonic stem cell lines derived from human blastocysts. Science. 1998;282:1145-7.

4. Takahashi K, Yamanaka S. Induction of pluripotent stem cells from mouse embryonic and adult fibroblast cultures by defined factors. Cell. 2006;126:663-76.

5. Takahashi K, et al. Induction of pluripotent stem cells from adult human fibroblasts by defined factors. Cell. 2007;131:861-72.

6. Cenini G, et al. Dissecting Alzheimer's disease pathogenesis in human 2D and 3D models. Mol Cell Neurosci. 2020;110:103568.

7. Karimian A, et al. CRISPR/Cas9 technology as a potent molecular tool for gene therapy. J Cell Physiol. 2019;234:12267-77.

8. Wang HX, et al. CRISPR/Cas9-based genome editing for disease modeling and therapy: challenges and opportunities for nonviral delivery. Chem Rev. 2017;117:9874-906. 
9. Zhao G, Pu J, Tang B. Applications of ZFN, TALEN and CRISPR/Cas9 techniques in disease modeling and gene therapy. Zhonghua Yi Xue Yi Chuan Xue Za Zhi. 2016;33:857-62.

10. Sterneckert JL, Reinhardt P, Scholer HR. Investigating human disease using stem cell models. Nat Rev Genet. 2014;15:625-39.

11. Lancaster MA, et al. Cerebral organoids model human brain development and microcephaly. Nature. 2013;501:373-9.

12. Quadrato $G$, et al. Cell diversity and network dynamics in photosensitive human brain organoids. Nature. 2017:545:48-53.

13. Arlotta P, Pasca SP. Cell diversity in the human cerebral cortex: from the embryo to brain organoids. Curr Opin Neurobiol. 2019;56:194-8.

14. Velasco $S$, et al. Individual brain organoids reproducibly form cell diversity of the human cerebral cortex. Nature. 2019;570:523-7.

15. Chen $\mathrm{HI}$, Song H, Ming GL. Applications of human brain organoids to clinical problems. Dev Dyn . 2019;248:53-64.

16. Amin ND, Pasca SP. Building models of brain disorders with threedimensional organoids. Neuron. 2018;100:389-405.

17. Eiraku M, Sasai Y. Self-formation of layered neural structures in threedimensional culture of ES cells. Curr Opin Neurobiol. 2012;22:768-77.

18. Eiraku $\mathrm{M}$, et al. Self-organized formation of polarized cortical tissues from ESCs and its active manipulation by extrinsic signals. Cell Stem Cell. 2008;3:519-32.

19. Lancaster MA, Knoblich JA. Generation of cerebral organoids from human pluripotent stem cells. Nat Protoc. 2014;9:2329-40.

20. Lancaster MA, Knoblich JA. Organogenesis in a dish: modeling development and disease using organoid technologies. Science. 2014;345:1247125.

21. Qian X, et al. Brain-region-specific organoids using mini-bioreactors for modeling ZIKV exposure. Cell. 2016;165:1238-54.

22. Qian X, et al. Sliced human cortical organoids for modeling distinct cortical layer formation. Cell Stem Cell. 2020;26:766-781.e769.

23. Sloan SA, et al. Human astrocyte maturation captured in 3D cerebral cortical spheroids derived from pluripotent stem cells. Neuron. 2017:95:779-790.e776.

24. Marton RM, et al. Differentiation and maturation of oligodendrocytes in human three-dimensional neural cultures. Nat Neurosci. 2019;22:484-91.

25. Camp JG, et al. Human cerebral organoids recapitulate gene expression programs of fetal neocortex development. Proc Natl Acad Sci USA. 2015;112:15672-7.

26. Renner $\mathrm{M}$, et al. Self-organized developmental patterning and differentiation in cerebral organoids. EMBO J. 2017;36:1316-29.

27. Mansour AA, et al. An in vivo model of functional and vascularized human brain organoids. Nat Biotechnol. 2018;36:432-41.

28. Koo B, Choi B, Park H, Yoon KJ. Past, present, and future of brain organoid technology. Mol Cells. 2019;42:617-27.

29. Qian X, et al. Generation of human brain region-specific organoids using a miniaturized spinning bioreactor. Nat Protoc. 2018;13:565-80.

30. Muguruma K, Nishiyama A, Kawakami H, Hashimoto K, Sasai Y. Selforganization of polarized cerebellar tissue in 3D culture of human pluripotent stem cells. Cell Rep. 2015;10:537-50.

31. Sakaguchi H, et al. Generation of functional hippocampal neurons from self-organizing human embryonic stem cell-derived dorsomedial telencephalic tissue. Nat Commun. 2015;6:8896.

32. Pellegrini $L$, et al. Human CNS barrier-forming organoids with cerebrospinal fluid production. Science. 2020. https://doi.org/10.1126/science. aaz5626.

33. Xiang Y, et al. Fusion of regionally specified hPSC-derived organoids models human brain development and interneuron migration. Cell Stem Cell. 2017;21:383-398.e387.

34. Xiang Y, et al. hESC-derived thalamic organoids form reciprocal projections when fused with cortical organoids. Cell Stem Cell. 2019;24:487497.e487.

35. Miura Y, et al. Generation of human striatal organoids and corticostriatal assembloids from human pluripotent stem cells. Nat Biotechnol. 2020;38:1421-30.

36. Sloan SA, Andersen J, Pasca AM, Birey F, Pasca SP. Generation and assembly of human brain region-specific three-dimensional cultures. Nat Protoc. 2018;13:2062-85.

37. Birey F, et al. Assembly of functionally integrated human forebrain spheroids. Nature. 2017;545:54-9.
38. Bagley JA, Reumann D, Bian S, Levi-Strauss J, Knoblich JA. Fused cerebral organoids model interactions between brain regions. Nat Methods. 2017;14:743-51.

39. Giandomenico SL, et al. Cerebral organoids at the air-liquid interface generate diverse nerve tracts with functional output. Nat Neurosci. 2019;22:669-79.

40. Andersen J, et al. Generation of functional human 3D cortico-motor assembloids. Cell. 2020;183:1913-1929.e1926.

41. Marton RM, Pasca SP. Organoid and assembloid technologies for investigating cellular crosstalk in human brain development and disease. Trends Cell Biol. 2020;30:133-43.

42. Pasca SP. Assembling human brain organoids. Science. 2019;363:126-7.

43. Chen A, Guo Z, Fang L, Bian S. Application of fused organoid models to study human brain development and neural disorders. Front Cell Neurosci. 2020;14:133.

44. Levitt P, Veenstra-VanderWeele J. Neurodevelopment and the origins of brain disorders. Neuropsychopharmacol. 2015;40:1-3.

45. Mitchell KJ. The genetics of neurodevelopmental disease. Curr Opin Neurobiol. 2011;21:197-203.

46. Gabriel E, Ramani A, Altinisik N, Gopalakrishnan J. Human Brain Organoids to Decode Mechanisms of Microcephaly. Front Cell Neurosci. 2020;14:115.

47. Fietz SA, et al. OSVZ progenitors of human and ferret neocortex are epithelial-like and expand by integrin signaling. Nat Neurosci. 2010;13:690-9.

48. Hansen DV, Lui JH, Parker PR, Kriegstein AR. Neurogenic radial glia in the outer subventricular zone of human neocortex. Nature. 2010;464:554-61.

49. Wang X, Tsai JW, LaMonica B, Kriegstein AR. A new subtype of progenitor cell in the mouse embryonic neocortex. Nat Neurosci. 2011:14:555-61.

50. Kanton $\mathrm{S}$, et al. Organoid single-cell genomic atlas uncovers humanspecific features of brain development. Nature. 2019;574:418-22.

51. Gabriel E, et al. CPAP promotes timely cilium disassembly to maintain neural progenitor pool. EMBO J. 2016;35:803-19.

52. Li R, et al. Recapitulating cortical development with organoid culture in vitro and modeling abnormal spindle-like (ASPM related primary) microcephaly disease. Protein Cell. 2017;8:823-33.

53. Ramani A, et al. Plk1/Polo phosphorylates Sas-4 at the onset of mitosis for an efficient recruitment of pericentriolar material to centrosomes. Cell Rep. 2018;25:3618-3630.e3616.

54. Zheng $X$, et al. Conserved TCP domain of Sas-4/CPAP is essential for pericentriolar material tethering during centrosome biogenesis. Proc Natl Acad Sci USA. 2014;111:E354-363.

55. Avidor-Reiss T, Gopalakrishnan J. Building a centriole. Curr Opin Cell Biol. 2013;25:72-7.

56. Gopalakrishnan J, et al. Sas-4 provides a scaffold for cytoplasmic complexes and tethers them in a centrosome. Nat Commun. 2011;2:359.

57. Lawo S, Hasegan M, Gupta GD, Pelletier L. Subdiffraction imaging of centrosomes reveals higher-order organizational features of pericentriolar material. Nat Cell Biol. 2012:14:1148-58.

58. Shohayeb B, et al. The association of microcephaly protein WDR62 with CPAP/IFT88 is required for cilia formation and neocortical development. Hum Mol Genet. 2020;29:248-63.

59. Zhang W, et al. Modeling microcephaly with cerebral organoids reveals a WDR62-CEP170-KIF2A pathway promoting cilium disassembly in neural progenitors. Nat Commun. 2019;10:2612.

60. Wang $L$, et al. Loss of NARS1 impairs progenitor proliferation in cortical brain organoids and leads to microcephaly. Nat Commun. 2020;11:4038.

61. Esk C, et al. A human tissue screen identifies a regulator of ER secretion as a brain-size determinant. Science. 2020;370:935-41.

62. Cugola FR, et al. The Brazilian Zika virus strain causes birth defects in experimental models. Nature. 2016;534:267-71.

63. Ventura CV, Maia M, Bravo-Filho V, Gois AL, Belfort R Jr. Zika virus in Brazil and macular atrophy in a child with microcephaly. Lancet. 2016;387:228

64. Li C, et al. Zika virus disrupts neural progenitor development and leads to microcephaly in mice. Cell Stem Cell. 2016;19:672. 
65. Qian X, Nguyen HN, Jacob F, Song H, Ming GL. Using brain organoids to understand Zika virus-induced microcephaly. Development. 2017; 144:952-7.

66. Gabriel E, et al. Recent zika virus isolates induce premature differentiation of neural progenitors in human brain organoids. Cell Stem Cell. 2017;20:397-406.e395.

67. Yoon KJ, et al. Zika-virus-encoded NS2A disrupts mammalian cortical neurogenesis by degrading adherens junction proteins. Cell Stem Cell. 2017:21:349-358.e346.

68. Xu M, et al. Identification of small-molecule inhibitors of Zika virus infection and induced neural cell death via a drug repurposing screen. Nat Med. 2016;22:1101-7.

69. Zhou T, et al. High-content screening in hPSC-neural progenitors identifies drug candidates that inhibit zika virus infection in fetal-like organoids and adult brain. Cell Stem Cell. 2017;21:274-283.e275.

70. Watanabe $M$, et al. Self-organized cerebral organoids with humanspecific features predict effective drugs to combat zika virus infection. Cell Rep. 2017;21:517-32.

71. Xu YP, et al. Zika virus infection induces RNAi-mediated antiviral immunity in human neural progenitors and brain organoids. Cell Res. 2019;29:265-73.

72. Mantegazza M. Epilepsy: advances in genetics and pathophysiology. Neurosci Lett. 2018;667:1-3.

73. Myers KA, Johnstone DL, Dyment DA. Epilepsy genetics: current knowledge, applications, and future directions. Clin Genet 2019;95:95-111.

74. Holmes GL, Noebels JL. The epilepsy spectrum: targeting future research challenges. Cold Spring Harb Perspect Med. 2016;6:a028043.

75. European Chromosome 16 Tuberous Sclerosis C. Identification and characterization of the tuberous sclerosis gene on chromosome 16 Cell. 1993;75:1305-15.

76. van Slegtenhorst $\mathrm{M}$, et al. Identification of the tuberous sclerosis gene TSC1 on chromosome 9q34. Science. 1997:277:805-8.

77. Curatolo P, Moavero R, de Vries PJ. Neurological and neuropsychiatric aspects of tuberous sclerosis complex. Lancet Neurol. 2015;14:733-45.

78. Crino PB, Nathanson KL, Henske EP. The tuberous sclerosis complex. N Engl J Med. 2006;355:1345-56.

79. Blair JD, Hockemeyer D, Bateup HS. Genetically engineered human cortical spheroid models of tuberous sclerosis. Nat Med. 2018;24:1568-78.

80. Splawski l, et al. Ca(V)12 calcium channel dysfunction causes a multisystem disorder including arrhythmia and autism. Cell. 2004:119:19-31.

81. Sharma SR, Gonda X, Tarazi Fl. Autism spectrum disorder: classification, diagnosis and therapy. Pharmacol Ther. 2018;190:91-104.

82. Jamain $\mathrm{S}$, et al. Reduced social interaction and ultrasonic communication in a mouse model of monogenic heritable autism. Proc Natl Acad Sci USA. 2008;105:1710-5.

83. Mariani J, et al. FOXG1-dependent dysregulation of GABA/glutamate neuron differentiation in autism spectrum disorders. Cell. 2015;162:375-90.

84. Wang P, et al. CRISPR/Cas9-mediated heterozygous knockout of the autism gene CHD8 and characterization of its transcriptional networks in neurodevelopment. Mol Autism. 2015:6:55.

85. Wang P, et al. CRISPR/Cas9-mediated heterozygous knockout of the autism gene CHD8 and characterization of its transcriptional networks in cerebral organoids derived from iPS cells. Mol Autism. 2017:8:11.

86. Woodbury-Smith $M$, et al. Mutations in RAB39B in individuals with intellectual disability, autism spectrum disorder, and macrocephaly. Mol Autism. 2017;8:59.

87. Zhang W, et al. Cerebral organoid and mouse models reveal a RAB39bPI3K-mTOR pathway-dependent dysregulation of cortical development leading to macrocephaly/autism phenotypes. Genes Dev. 2020;34:580-97.

88. Dawson TM, Ko HS, Dawson VL. Genetic animal models of Parkinson's disease. Neuron. 2010;66:646-61.

89. Laurijssens B, Aujard F, Rahman A. Animal models of Alzheimer's disease and drug development. Drug Discov Today Technol. 2013;10:e319-327.

90. Ribeiro FM, Camargos ER, de Souza LC, Teixeira AL. Animal models of neurodegenerative diseases. Rev Bras Psiquiatr. 2013;35(Suppl 2):S82-91.
91. Querfurth HW, LaFerla FM. Alzheimer's disease. N Engl J Med. 2010;362:329-44.

92. Long JM, Holtzman DM. Alzheimer disease: an update on pathobiology and treatment strategies. Cell. 2019;179:312-39.

93. Duyckaerts C, Potier MC, Delatour B. Alzheimer disease models and human neuropathology: similarities and differences. Acta Neuropathol. 2008;115:5-38.

94. Ghatak S, et al. Mechanisms of hyperexcitability in Alzheimer's disease hiPSC-derived neurons and cerebral organoids vs isogenic controls. Elife. 2019. https://doi.org/10.7554/eLife.50333.

95. Gonzalez C, et al. Modeling amyloid beta and tau pathology in human cerebral organoids. Mol Psychiatry. 2018;23:2363-74.

96. Lin YT, et al. APOE4 causes widespread molecular and cellular alterations associated with alzheimer's disease phenotypes in human iPSCderived brain cell types. Neuron. 2018;98:1141-1154.e1147.

97. Zhao J, et al. APOE4 exacerbates synapse loss and neurodegeneration in Alzheimer's disease patient iPSC-derived cerebral organoids. Nat Commun. 2020;11:5540.

98. Perez MJ, et al. Loss of function of the mitochondrial peptidase PITRM1 induces proteotoxic stress and Alzheimer's disease-like pathology in human cerebral organoids. Mol Psychiatry. 2020. https://doi.org/10. 1038/s41380-020-0807-4

99. Wang Z, et al. BACE2, a conditional beta-secretase, contributes to Alzheimer's disease pathogenesis. JCl Insight. 2019. https://doi.org/10. 1172/jci.insight.123431.

100. Voytyuk $I$, et al. BACE2 distribution in major brain cell types and identification of novel substrates. Life Sci Alliance. 2018;1:e201800026.

101. Dominguez D, et al. Phenotypic and biochemical analyses of BACE1and BACE2-deficient mice. J Biol Chem. 2005:280:30797-806.

102. Alic I, et al. Patient-specific Alzheimer-like pathology in trisomy 21 cerebral organoids reveals BACE2 as a gene dose-sensitive AD suppressor in human brain. Mol Psychiatry. 2020. https://doi.org/10.1002/alz. 043136.

103. Park JC, et al. A logical network-based drug-screening platform for Alzheimer's disease representing pathological features of human brain organoids. Nat Commun. 2021;12:280.

104. Weintraub D, Burn DJ. Parkinson's disease: the quintessential neuropsychiatric disorder. Mov Disord. 2011;26:1022-31.

105. Tieng $V$, et al. Engineering of midbrain organoids containing long-lived dopaminergic neurons. Stem Cells Dev. 2014:23:1535-47.

106. Monzel AS, et al. Derivation of human midbrain-specific organoids from neuroepithelial stem cells. Stem Cell Rep. 2017:8:1144-54.

107. Jo J, et al. Midbrain-like organoids from human pluripotent stem cells contain functional dopaminergic and neuromelanin-producing neurons. Cell Stem Cell. 2016;19:248-57.

108. KwakTH, et al. Generation of homogeneous midbrain organoids with in vivo-like cellular composition facilitates neurotoxin-based Parkinson's disease modeling. Stem cells. 2020;38:727-40.

109. Kluss JH, Mamais A, Cookson MR. LRRK2 links genetic and sporadic Parkinson's disease. Biochem Soc Trans. 2019;47:651-61.

110. Kim H, et al. Modeling G2019S-LRRK2 sporadic parkinson's disease in 3D midbrain organoids. Stem Cell Rep. 2019;12:518-31.

111. Smits LM, et al. Modeling Parkinson's disease in midbrain-like organoids. NPJ Parkinson's Dis. 2019;5:5.

112. Walker FO. Huntington's disease. Semin Neurol. 2007;27:143-50.

113. Potter NT, Spector EB, Prior TW. Technical standards and guidelines for Huntington disease testing. Genet Med . 2004;6:61-5.

114. Conforti $P$, et al. Faulty neuronal determination and cell polarization are reverted by modulating HD early phenotypes. Proc Natl Acad Sci USA. 2018:115:E762-71.

115. Crespo M, et al. Colonic organoids derived from human induced pluripotent stem cells for modeling colorectal cancer and drug testing. Nat Med. 2017;23:878-84.

116. Bian $\mathrm{S}$, et al. Genetically engineered cerebral organoids model brain tumor formation. Nat Methods. 2018;15:631-9.

117. Saengwimol D, et al. A three-dimensional organoid model recapitulates tumorigenic aspects and drug responses of advanced human retinoblastoma. Sci Rep. 2018;8:15664. 
118. Dost AFM, et al. Organoids model transcriptional hallmarks of oncogenic KRAS activation in lung epithelial progenitor cells. Cell Stem Cell. 2020;27:663-67.e668.

119. Breunig $M$, et al. Modeling plasticity and dysplasia of pancreatic ductal organoids derived from human pluripotent stem cells. Cell Stem Cell. 2021. https://doi.org/10.1016/j.stem.2021.03.005.

120. Huang $L$, et al. Commitment and oncogene-induced plasticity of human stem cell-derived pancreatic acinar and ductal organoids. Cell Stem Cell. 2021. https://doi.org/10.1016/j.stem.2021.03.022.

121. Ferreira RMM, et al. Duct- and acinar-derived pancreatic ductal adenocarcinomas show distinct tumor progression and marker expression. Cell Rep. 2017;21:966-78.

122. Lee $A Y L$, et al. Cell of origin affects tumour development and phenotype in pancreatic ductal adenocarcinoma. Gut. 2019;68:487-98.

123. Stupp R, et al. Radiotherapy plus concomitant and adjuvant temozolomide for glioblastoma. N Engl J Med. 2005;352:987-96.

124. Roy S, Lahiri D, Maji T, Biswas J. Recurrent glioblastoma: where we stand. South Asian J Cancer. 2015:4:163-73.

125. Kreis। TN, et al. Phase II trial of single-agent bevacizumab followed by bevacizumab plus irinotecan at tumor progression in recurrent glioblastoma. JCO . 2009;27:740-5.

126. Neftel $C$, et al. An integrative model of cellular states, plasticity, and genetics for glioblastoma. Cell. 2019;178:835-849.e821.

127. Brennan CW, et al. The somatic genomic landscape of glioblastoma. Cell. 2013;155:462-77.

128. Mariappan A, Goranci-Buzhala G, Ricci-Vitiani L, Pallini R, Gopalakrishnan J. Trends and challenges in modeling glioma using 3D human brain organoids. Cell Death Differ. 2021;28:15-23.

129. Azzarelli R. Organoid models of glioblastoma to study brain tumor stem cells. Front Cell Dev Biol. 2020;8:220.

130. Sturm $D$, et al. New brain tumor entities emerge from molecular classification of CNS-PNETs. Cell. 2016;164:1060-72.

131. Momota H, Shih AH, Edgar MA, Holland EC. c-Myc and beta-catenin cooperate with loss of p53 to generate multiple members of the primitive neuroectodermal tumor family in mice. Oncogene. 2008:27:4392-401.

132. Ogawa J, Pao GM, Shokhirev MN, Verma IM. Glioblastoma model using human cerebral organoids. Cell Rep. 2018;23:1220-9.

133. da Silva B, Mathew RK, Polson ES, Williams J, Wurdak H. Spontaneous glioblastoma spheroid infiltration of early-stage cerebral organoids models brain tumor invasion. SLAS Discov . 2018;23:862-8.

134. Linkous $A$, et al. Modeling patient-derived glioblastoma with cerebral organoids. Cell Rep. 2019;26:3203-3211.e3205.

135. Goranci-Buzhala G, et al. Rapid and efficient invasion assay of glioblastoma in human brain organoids. Cell Rep. 2020;31:107738.

136. Lancaster MA, et al. Guided self-organization and cortical plate formation in human brain organoids. Nat Biotechnol. 2017;35:659-66.
137. Yoon SJ, et al. Reliability of human cortical organoid generation. Nat Methods. 2019;16:75-8.

138. Zhu Y, et al. In situ generation of human brain organoids on a micropillar array. Lab Chip. 2017;17:2941-50.

139. Giandomenico SL, Sutcliffe M, Lancaster MA. Generation and long-term culture of advanced cerebral organoids for studying later stages of neural development. Nat Protoc. 2021;16:579-602.

140. Pham MT, et al. Generation of human vascularized brain organoids. NeuroReport. 2018:29:588-93.

141. Ham O, Jin YB, Kim J, Lee MO. Blood vessel formation in cerebral organoids formed from human embryonic stem cells. Biochem Biophys Res Commun. 2020:521:84-90.

142. Cakir B, et al. Engineering of human brain organoids with a functional vascular-like system. Nat Methods. 2019;16:1169-75.

143. Shi Y, et al. Vascularized human cortical organoids (vOrganoids) model cortical development in vivo. PLoS Biol. 2020;18:e3000705.

144. Stebbins MJ, et al. Human pluripotent stem cell-derived brain pericyte-like cells induce blood-brain barrier properties. Sci Adv . 2019;5:eaau7375.

145. Campisi M, et al. 3D self-organized microvascular model of the human blood-brain barrier with endothelial cells, pericytes and astrocytes. Biomaterials. 2018;180:117-29.

146. Vatine GD, et al. Human iPSC-derived blood-brain barrier chips enable disease modeling and personalized medicine applications. Cell Stem Cell. 2019;24:995-1005.e1006.

147. Canfield SG, et al. An isogenic blood-brain barrier model comprising brain endothelial cells, astrocytes, and neurons derived from human induced pluripotent stem cells. J Neurochem. 2017;140:874-88.

148. Konishi $\mathrm{H}$, Kiyama $\mathrm{H}$, Ueno M. Dual functions of microglia in the formation and refinement of neural circuits during development. Int J Dev Neurosci . 2019:77:18-25.

149. Gutmann DH, Kettenmann H. Microglia/brain macrophages as central drivers of brain tumor pathobiology. Neuron. 2019;104:442-9.

150. Beggs S, Salter MW. SnapShot: microglia in disease. Cell. 2016;165:12941294.e1291.

151. Ormel PR, et al. Microglia innately develop within cerebral organoids. Nat Commun. 2018:9:4167.

152. Song $\mathrm{L}$, et al. Functionalization of brain region-specific spheroids with isogenic microglia-like cells. Sci Rep. 2019;9:11055.

153. Bejoy J, et al. Genomics analysis of metabolic pathways of human stem cell-derived microglia-like cells and the integrated cortical spheroids. Stem Cells Int. 2019;2019:2382534.

\section{Publisher's Note}

Springer Nature remains neutral with regard to jurisdictional claims in published maps and institutional affiliations.
Ready to submit your research? Choose BMC and benefit from:

- fast, convenient online submission

- thorough peer review by experienced researchers in your field

- rapid publication on acceptance

- support for research data, including large and complex data types

- gold Open Access which fosters wider collaboration and increased citations

- maximum visibility for your research: over $100 \mathrm{M}$ website views per year

At $\mathrm{BMC}$, research is always in progress.

Learn more biomedcentral.com/submissions 